\title{
Use of a glycerol-limited, long-term chemostat for isolation of Escherichia coli mutants with improved physiological properties
}

\author{
Christian Weikert, Uwe Sauer and James E. Bailey
}

Author for correspondence: James E. Bailey. Tel: +411633 3170. Fax: +4116331051.

Institute of Biotechnology, ETH Zürich, $\mathrm{CH}-8093$

Zürich, Switzerland

\begin{abstract}
The evolution of Escherichia coli MG1655 mutants was followed over $126 \mathrm{~d}$ in a glycerol-limited chemostat at a dilution rate of $0.05 \mathrm{~h}^{-1}$. This corresponds to a total of 217 generations at a doubling time of $13.9 \mathrm{~h}$. After this time, nearly $90 \%$ of the chemostat population consisted of evolved mutant strains as determined by their altered colony morphologies on plates. Two mutants were isolated that exhibited generally improved growth phenotypes in batch cultivations on glycerol, glucose or the gluconeogenic substrate acetate. Higher specific growth rates and increased biomass yields were found for both mutants. For one mutant, this behaviour was combined with significantly reduced secretion of overflow metabolites when either glycerol or glucose was the carbon source. Additionally, during all growth phases of a batch cultivation, this mutant exhibited increased resistance to a variety of adverse conditions including heat shock, osmotic stress and nutrient deprivation. It also displayed significantly shorter lag phases.
\end{abstract}

Keywords: Escherichia coli, growth physiology, chemostat selection, stress resistance, glycerol limitation

\section{INTRODUCTION}

It is well known that, when propagated in fixed environments, microbial populations adapt to these conditions by mutation and natural selection, and that the power of continuous cultivation is useful for studying such evolution processes (Novick \& Szilard, 1950). In particular, when studied over prolonged periods, mutants with selective advantages will eventually replace the parental strains in these dynamic chemostat populations (Moser, 1958). Subsequently, chemostats have been used to isolate mutants with improved or new metabolic capabilities (Cheng et al., 1989; Dykhuizen \& Hartl, 1983) or to study evolution (Dykhuizen \& Hartl, 1981; Helling et al., 1987; Paquin \& Adams, 1983). Adaptive mutations that appear under substrate-limited conditions during continuous cultivation have been reported to result in increased maximum specific growth rates $\left(\mu_{\max }\right)$ and/or higher affinity for the limiting substrate, which can be interpreted as a reduction in the value of the Monod kinetics halfsaturation constant $K_{\mathrm{s}}$ (Dykhuizen \& Hartl, 1983; Kurland, 1992).

An important phenomenon concerning the clone-specific metabolism in such adapted populations is the frequent observance of co-metabolism, manifested as a morphological and physiological polymorphism within the population. This co-metabolism is mainly established by a higher substrate uptake rate and the subsequent secretion of incompletely oxidized metabolites by some mutants combined with the efficient utilization of these compounds by other, minority, clones. For example, Escherichia coli cultures in a glucose-limited chemostat, starting with a single clone, result in a polymorphic population, in which several distinct mutant strains coexist (Dykhuizen \& Hartl, 1981; Helling et al., 1987; Rosenzweig et al., 1994). Around $60 \%$ of the chemostat population may consist of mutants with a metabolite secretion phenotype (Helling et al., 1987). As a mixed population, these cultures outcompete the parental strain. However, under batch growth conditions, individual mutants may show growth characteristics that are not improved or are even inferior compared to the parental strain.

The control of substrate uptake and catabolism in the absence of growth is a key to improving the productivity and operational life-span of microbial biocatalysts in biotechnological applications (Matin, 1992). For optimal exploitation of microbial metabolic abilities, process organisms that express their potential catabolic rates under slow or non-growing conditions and yet maintain a tight control of overflow metabolism are 
desirable. A selection approach aimed at the development of strains with generally enhanced carbon utilization efficiency under slow-growth conditions should be characterized by low growth rates and conditions that minimize the likelihood that co-metabolism will be beneficial. Although strains with improved growth characteristics have been selected before, these selection experiments were usually performed at intermediate dilution rates, in order to study evolution and population dynamics, during many generations, more effectively (Dykhuizen \& Hartl, 1983; Helling et al., 1987). Also, glucose was used as the carbon source in these studies, but it is not the preferred substrate in a selection approach that aims at the development of a single clone with 'improved' characteristics, as it selects for efficient co-metabolism (Rosenzweig et al., 1994). An additional drawback of glucose as the limiting substrate is the reported suppression of mutations in glucosegrown cultures (MacPhee, 1993). As an alternative substrate, glycerol is potentially of interest as it can circumvent both problems to a certain extent (MacPhee, 1993; Sakamoto et al., 1996). We are not aware of any mutant described in the literature that exhibits both an improved biomass yield and increased $\mu_{\max }$ phenotype on several different substrates coupled with lower excretion of overflow metabolites and generally improved stability in harsh environments such as carbon starvation.

Here we describe the evolution of an E. coli population from a single clone in a glycerol-limited chemostat. The continuous culture was maintained at a low dilution rate of $0.05 \mathrm{~h}^{-1}$ over a period of $126 \mathrm{~d}$. Individual clones were analysed for growth characteristics and two significantly 'improved' strains were characterized in detail with respect to their growth physiology on various substrates and their stress resistance.

\section{METHODS}

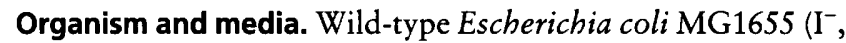
$\mathrm{F}^{-}$, CSCG 6300; Bachmann, 1987) was used for the selection experiment. Stock cultures were maintained at $-70^{\circ} \mathrm{C}$ in $30 \%(\mathrm{v} / \mathrm{v})$ glycerol. If not stated otherwise, a modified, defined M9 medium containing the following components was used for cultivations: $30 \mathrm{mM}\left(\mathrm{NH}_{4}\right)_{2} \mathrm{SO}_{4}, 6.6 \mathrm{mM} \mathrm{K}_{2} \mathrm{HPO}_{4}$, $3.7 \mathrm{mM} \mathrm{KH}_{2} \mathrm{PO}_{4}, 1 \mathrm{mM} \mathrm{MgSO}{ }_{4}, 50 \mu \mathrm{M} \mathrm{CaCl}_{2}, 0.2 \mathrm{mM} \mathrm{FeCl}_{2}$ and $0.3 \mu \mathrm{M}$ vitamin $\mathrm{B}_{1}$. Polypropylene glycol 2000 (Fluka) was added at $100 \mu \mathrm{ll}^{-1}$ to prevent foaming in bioreactor fermentations. Concentrated stock solutions of carbon sources $50 \%(\mathrm{w} / \mathrm{v})$ glucose, $50 \%(\mathrm{v} / \mathrm{v})$ glycerol and $4 \mathrm{M}$ sodium acetate $(\mathrm{pH} 7 \cdot 0)$ - were prepared and autoclaved separately at $121^{\circ} \mathrm{C}$ for $20 \mathrm{~min}$.

Chemostat culture. Continuous culture for selection was carried out in a 1.51 bench-top fermenter (Bioengineering AG) with a working volume of $1.0 \mathrm{l}$ and a dilution rate of $0.05 \mathrm{~h}^{-1}$. The cultivation conditions were: $\mathrm{pH} 7 \cdot 0 \pm 0 \cdot 1$ controlled by addition of $4 \mathrm{M} \mathrm{NaOH}$; temperature $37^{\circ} \mathrm{C}$; stirring at 1200 r.p.m.; aeration with filter-sterilized air was set to 1.25 v.v.m. (volume air $_{\text {volume }}$ liquid $^{-1} \mathrm{~min}^{-1}$ ). The culture volume was kept constant by a mass-controlled pump. The feed was M9 medium with $0.5 \%$ glycerol as the growth-limiting substrate. This was prepared in 201 batches and sterilized by filtration through a $0.2 \mu \mathrm{m}$ membrane filter. To avoid growth in the feeding line, a possibility at this low flow rate, the tubing between the fermenter and the trap was changed every 3 weeks. Excessive adherence of cells to the fermenter walls was prevented by increasing the impeller speed to 1700 r.p.m. for 5 min every second day. A single colony from a plate was used to inoculate an overnight culture in a shake flask, which was then used as the inoculum for the chemostat.

Batch cultivations. A bench-top Sixforce multi-bioreactor system (Infors), consisting of six reactors each with $350 \mathrm{ml}$ working volume, was used for the determination of batch growth characteristics. The cultivation conditions were: pH 7.0 \pm 0.1 controlled by addition of $4 \mathrm{M} \mathrm{NaOH}$ or $2 \mathrm{M}$ $\mathrm{H}_{3} \mathrm{PO}_{4}$; temperature $37^{\circ} \mathrm{C}$; stirring at 500 r.p.m.; aeration with filter-sterilized air at $475 \mathrm{ml} \mathrm{min}{ }^{-1}$. Fermenters containing basic medium and antifoam were sterilized by autoclaving at $121{ }^{\circ} \mathrm{C}$ for $30 \mathrm{~min}$. Sterile solutions of $\mathrm{MgSO}_{4}, \mathrm{CaCl}_{2}, \mathrm{FeCl}_{2}$ and the carbon source were added separately afterwards at $37^{\circ} \mathrm{C}$. Samples from the batch cultivations were collected by using the Sample Bus System (Biospectra AG) reactor interface. To quench further biological activity in the samples, an equal volume of stop solution at $0{ }^{\circ} \mathrm{C}$, containing $6 \mathrm{mM} \mathrm{NaN}_{3}$ was added automatically to the samples as they were collected.

Isolation of mutants. Samples from the chemostat were withdrawn at regular intervals and preserved as frozen stocks. To demonstrate that the ongoing cultivation was free of contaminants, diluted aliquots were plated on solidified LB medium. The same medium supplemented with $100 \mu \mathrm{g}$ ampicillin $\mathrm{ml}^{-1}$ was used to detect potential infection by Pseudomonas spp., which are frequently found as contaminants of $E$. coli cultivations, having a very similar colony morphology on non-selective plates. Over the entire period of 18 weeks, no contaminations were observed. To quantify the partition of the different colony morphologies in the culture, appropriate dilutions of frozen samples were plated on solidified LB and M9 medium to yield between 100 and 200 colonies per plate. After $3 \mathrm{~d}$ incubation at $37^{\circ} \mathrm{C}$, different colony morphologies were discernible, and they were counted. Control plates containing wild-type MG1655 showed homogeneous colony morphologies.

Analytical procedures. A Synchron CX5CE automated enzyme analysis system (Beckman) was used to determine glucose, glycerol and acetate concentrations using either kits from Beckman or reagents adapted from kits for acetate (Boehringer) and glycerol (Sigma) analysis. Reverse-phase HPLC was used to determine the concentrations of succinate, lactate and formate. A Merck Hitachi system equipped with a Nucleosil C8 reverse-phase column and $5 \mu \mathrm{m}$ particle size (Bischof Scientific Instruments) was used to separate the analytes. Absorption at $210 \mathrm{~nm}$ was used for detection. The mobile phase was $0.2 \mathrm{M} \mathrm{H}_{3} \mathrm{PO}_{4}$ at a flow rate of $0.7 \mathrm{ml} \mathrm{min}$. Cell growth during the batch cultivations was monitored as the $\mathrm{OD}_{420}$ of appropriate dilutions in $0.9 \%$ formaldehyde. For determination of cellular dry weight, a known volume of fermentation broth was pelleted down in pre-weighed glass tubes at 3000 r.p.m. at $4{ }^{\circ} \mathrm{C}$ for 10 min using a Beckman CS 6R centrifuge. The cells were washed once with PBS buffer $(0.145 \mathrm{M} \mathrm{NaCl} ; 0.15 \mathrm{M}$ sodium phosphate) and once with water. The tubes were dried at $90^{\circ} \mathrm{C}$ for $12-16 \mathrm{~h}$ to a constant weight. The fermenter off gas composition was determined with a Fisons quadrupole mass spectrometer (Fisons Instruments).

Determination of specific growth parameters. Data from the 
exponential growth phase of strains cultivated in the multireactor system were used to determine growth parameters on the different substrates. Maximum growth rates $\left(\mu_{\max }\right)$ were obtained as the slopes of the linear regression of semi-log time plots of the optical density. Biomass concentration was obtained from optical density data by previously determined linear relationships between optical density at $420 \mathrm{~nm}$ and cellular dry weight for each strain and carbon source. Polynomal interpolations of $\mathrm{OD}_{420}$ and substrate concentration data were used to calculate specific substrate uptake rates. Biomass yield data were obtained from exponential growth phase by dividing the slope of the determined growth rate by the substrate utilization rate.

Determination of lag phases. Inocula for the determination of the growth-phase-dependent lag phases were cultivated in M9 medium supplemented with $1 \%$ glucose and $1 \%$ LB. Washed cells from different growth phases were used as inocula for a growth assay in microtitre plates using the same medium. The assays were performed in 96-well plates in a Bio-Rad $3550 \mathrm{UV}$ Reader equipped with a Dualchannel incubator at $30^{\circ} \mathrm{C}$. The low temperature was chosen to minimize evaporation. $\mathrm{OD}_{595}$ was used to monitor the time course of growth. The initial composition of the medium in the assay was identical to the medium used for the batch cultivations. The end of lag phase was determined as the time at which the extrapolated exponential phase curve fit intersected the initial optical density.

Assessment of stress resistance. Resistance to heat shock, osmotic stress and nutrient deprivation was determined for cells harvested from both exponential and stationary phases. Stationary phase cells were harvested at least $12 \mathrm{~h}$ after entry into stationary phase. Cells were grown in shake flasks under conditions identical to those used for the determination of lag phases. Prior to all incubations, the cells were washed once with PBS. The heat-shocked cells were incubated with shaking at $55^{\circ} \mathrm{C}$ in prewarmed PBS in an Eppendorf 5436 Thermomixer. The resistance to high osmolarity was determined by incubation in PBS supplemented with $1.0 \mathrm{M} \mathrm{NaCl}$ at $37^{\circ} \mathrm{C}$. Survival under nutrient starvation was determined by incubating washed cell suspensions at $37^{\circ} \mathrm{C}$ in agitated shake flasks containing M9 medium without any carbon source. Appropriate dilutions of samples from the incubations in PBS were plated on solidified LB medium to yield between 50 and 400 colonies per plate. The reported viable cell numbers are the mean values of 16 plates per time point: four plates each from two independent dilutions from each of two parallel experiments.

\section{RESULTS}

\section{Selection of mutants}

Wild-type E. coli MG1655 was grown in a glycerollimited chemostat culture under aerobic conditions at a dilution rate of $0.05 \mathrm{~h}^{-1}$. Glycerol was used as the carbon source to avoid glucose repression of spontaneous mutagenesis (Dykhuizen \& Hartl, 1983; MacPhee, 1993) and to reduce the formation of overflow products (Sakamoto et al., 1996). Two different colony morphologies, clearly discernible from the morphology of the wild-type, were first identified after 4 weeks. Over the remaining cultivation period of 14 weeks, the relative proportion of cells with those morphologies increased continuously while the fraction of clones with the wild-

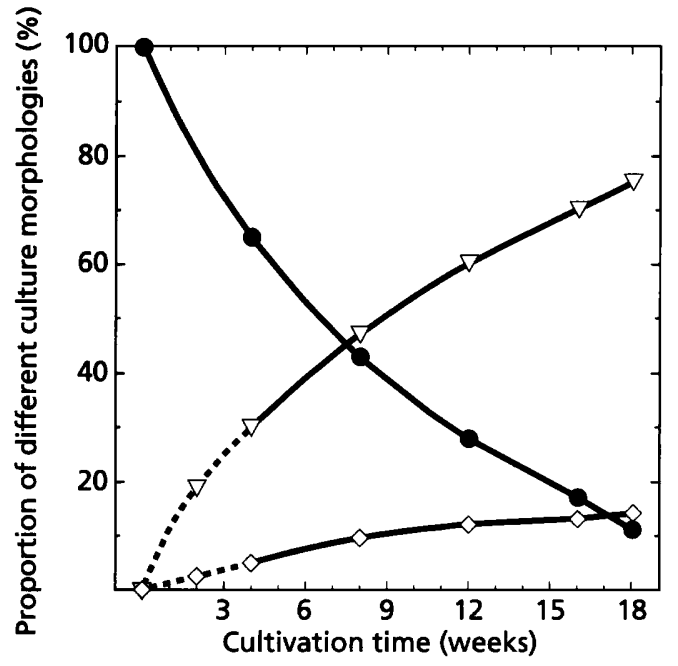

Fig. 1. Relative frequencies of different colony morphologies in the $E$. coli population in the glycerol-limited chemostat culture $\left(D=0.05 h^{-1}\right)$. The symbols indicate the fraction of cells with wild-type-like morphology $(\Theta)$, larger colonies $(\nabla)$ and smaller colonies $(\diamond)$. The values indicated are based on the counts from six plates per time point.

type-like colony morphology decreased (Fig. 1). The experiment was stopped when approximately $90 \%$ of the population consisted of cells with new colony morphologies. Cells displaying these two new morphologies comprised two groups of clones showing either significantly larger and irregular shaped (L) or much smaller ( $\mathrm{S}$ ) colonies as compared to wild-type when grown on LB plates. The proportion of the population forming larger colonies reached more than $75 \%$ of the total population by the end of the experiment (Fig. 1).

\section{Isolation and characterization of mutant strains}

Several clones of each morphology were isolated from plates and subjected to initial analysis in shake flask growth experiments on M9 minimal medium with glycerol as the carbon source. Isolates with morphology $S$ showed weak growth in liquid culture and increased adherence to the walls of the flasks compared to the wild-type. Therefore, clones with this morphology were not subjected to further analysis. Most isolates with morphology L exhibited higher growth rates and increased biomass yields under the conditions investigated. As typical representatives of morphology $\mathrm{L}$, the mutants CWML1 and CWML2 were selected for further, more detailed, characterization.

All clones stably maintained their altered morphologies on plates and their growth characteristics in liquid culture. No alteration of the phenotypes was observed after intervening batch cultivations. To ensure the close relationship of the isolated mutants to the $E$. coli parent strain (MG1655), protein analysis using two-dimen- 
Table 1. Batch growth characteristics of $E$. coli wild-type MG1655, mutant CWML1 and mutant CWML2 in bioreactor cultivations $\left(37^{\circ} \mathrm{C}, \mathrm{pH} 7.0 \pm 0.2\right.$, aeration 1.5 v.v.m.)

M9 minimal medium was used and supplemented with one of three different carbon sources: $0.5 \%$ glucose, $0.5 \%$ glycerol or $0.6 \%$ acetate. Maximum growth rates, indicated as $\mu_{\max }\left(\mathrm{h}^{-1}\right)$, were determined using linear regression of semi-log plots of $\mathrm{OD}_{420}$ versus time. Biomass yields were calculated during exponential growth phase. The yields of the wild-type were $0.519 \mathrm{~g} \mathrm{~g}^{-1}$ on glycerol, $0.316 \mathrm{~g} \mathrm{~g}^{-1}$ on acetate and $0.419 \mathrm{~g} \mathrm{~g}^{-1}$ on glucose. The values represented are all based on at least three independent cultivations.

\begin{tabular}{|c|c|c|c|c|}
\hline \multirow[t]{2}{*}{ Carbon source } & \multirow[t]{2}{*}{ Growth parameter } & \multicolumn{3}{|c|}{ Strain } \\
\hline & & $\begin{array}{l}\text { Wild-type } \\
\text { (MG1655) }\end{array}$ & $\begin{array}{l}\text { Mutant } \\
\text { CWML1 }\end{array}$ & $\begin{array}{l}\text { Mutant } \\
\text { CWML2 }\end{array}$ \\
\hline Glycerol & $\begin{array}{l}\mu_{\max }\left(\mathrm{h}^{-1}\right) \\
\text { Relative biomass yield }(\%) \\
\text { Maximum acetate concn }(\mathrm{mM})\end{array}$ & $\begin{array}{l}0 \cdot 32 \pm 0 \cdot 02 \\
100 \pm 3 \\
2 \cdot 7 \pm 0 \cdot 2\end{array}$ & $\begin{array}{l}0 \cdot 33 \pm 0 \cdot 02 \\
108 \pm 2 \\
2 \cdot 4 \pm 0 \cdot 2\end{array}$ & $\begin{array}{l}0 \cdot 39 \pm 0 \cdot 02 \\
113 \pm 2 \\
0 \cdot 9 \pm 0 \cdot 1\end{array}$ \\
\hline Acetate & $\begin{array}{l}\mu_{\max }\left(\mathrm{h}^{-1}\right) \\
\text { Relative biomass yield }(\%)\end{array}$ & $\begin{array}{l}0 \cdot 22 \pm 0 \cdot 01 \\
100 \pm 2\end{array}$ & $\begin{array}{l}0 \cdot 23 \pm 0 \cdot 01 \\
116 \pm 2\end{array}$ & $\begin{array}{l}0 \cdot 26 \pm 0 \cdot 02 \\
123 \pm 3\end{array}$ \\
\hline Glucose & $\begin{array}{l}\mu_{\max }\left(\mathrm{h}^{-1}\right) \\
\text { Relative biomass yield }(\%) \\
\text { Maximum acetate concn }(\mathrm{mM}) \\
\text { Maximum lactate concn }(\mathrm{mM}) \\
\text { Maximum succinate concn }(\mathrm{mM}) \\
\mathrm{CO}_{2} \text { production rate }[\mathrm{mmol} \\
\left.(\mathrm{g} \text { dry } \mathrm{wt})^{-1} \mathrm{~h}^{-1}\right]\end{array}$ & $\begin{array}{l}0.52 \pm 0.05 \\
100 \pm 4 \\
3 \cdot 2 \pm 0 \cdot 1 \\
0 \cdot 30 \pm 0 \cdot 02 \\
0 \cdot 12 \pm 0 \cdot 01 \\
13 \cdot 2 \pm 2\end{array}$ & $\begin{array}{c}0.64 \pm 0.03 \\
114 \pm 2 \\
3 \cdot 1 \pm 0 \cdot 1 \\
0 \cdot 23 \pm 0 \cdot 02 \\
0 \cdot 12 \pm 0 \cdot 02 \\
11 \cdot 2 \pm 0.6\end{array}$ & $\begin{array}{c}0.67 \pm 0.03 \\
121 \pm 3 \\
2 \cdot 6 \pm 0 \cdot 10 \\
0 \cdot 16 \pm 0 \cdot 02 \\
0 \cdot 20 \pm 0 \cdot 02 \\
9 \cdot 1 \pm 1\end{array}$ \\
\hline
\end{tabular}

sional gel electrophoresis was employed. A very high degree of similarity at the level of detected protein proved their descendance from MG1655 (approximately 1200 identical spots out of a total of about 1300 detected).

\section{Batch cultivations}

To quantify the batch growth characteristics, the parental strain MG1655 and mutants CWML1 and CWML2 were grown in controlled bioreactors using M9 medium supplemented with $0.5 \%$ glycerol, as in the selection experiment. Both mutant strains showed markedly increased biomass yields on this substrate, and mutant CWML2 exhibited a significantly higher growth rate compared to the wild-type (Table 1 ). The maximum concentration of the overflow metabolite acetate accumulated by this mutant strain during batch growth was only a fraction of that produced by the wild-type (Fig. 2) and by mutant CWML1 (Table 1). After consumption of acetate, the only carbon source present at this stage of the cultivation, an increase in $\mathrm{OD}_{420}$ without increase in cell number was observed for both the wild-type and the mutants, apparently as a consequence of the transition to stationary phase. To distinguish potential substratespecific phenotypes from general growth characteristics, batch cultivations were also performed with glucose and the gluconeogenic substrate acetate as carbon sources. As on glycerol, both mutants CWML1 and CWML2 exhibited generally increased biomass yields on these

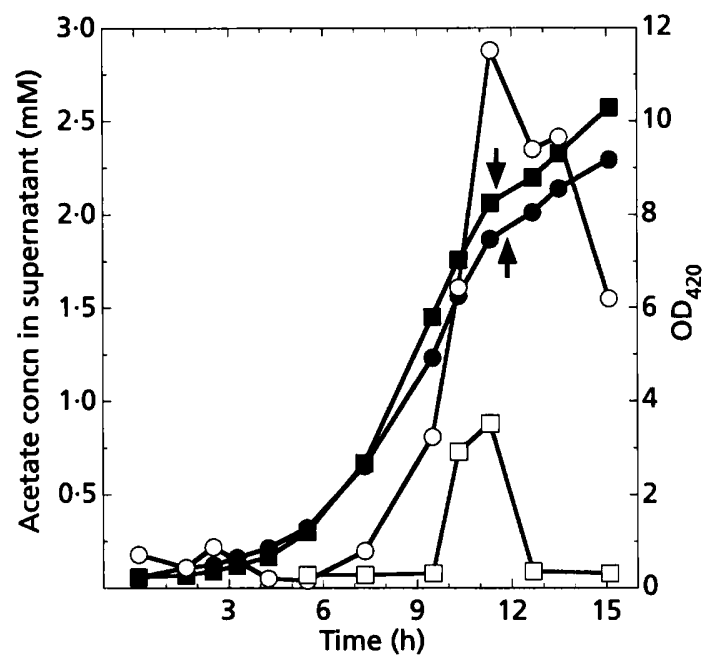

Fig. 2. Growth behaviour of $E$. coli MG1655 and mutant CWML2 in M9 medium containing $0.5 \%$ glycerol as the carbon source $\left(37^{\circ} \mathrm{C}, \mathrm{pH} 7.0 \pm 0.2\right.$, aeration 1.5 v.v.m.). $\mathrm{OD}_{420}$ of wildtype (O) and mutant CWML2 ( $\square$ ) is shown on the right axis; the left axis shows the acetate concentration in the supernatants of wild-type $(O)$ and mutant $(\square)$. Arrows indicate the time of complete depletion of glycerol for each strain.

substrates (Table 1). In particular, mutant CWML2 grew significantly faster than the wild-type on all substrates investigated. Also, reduced acetate overflow 


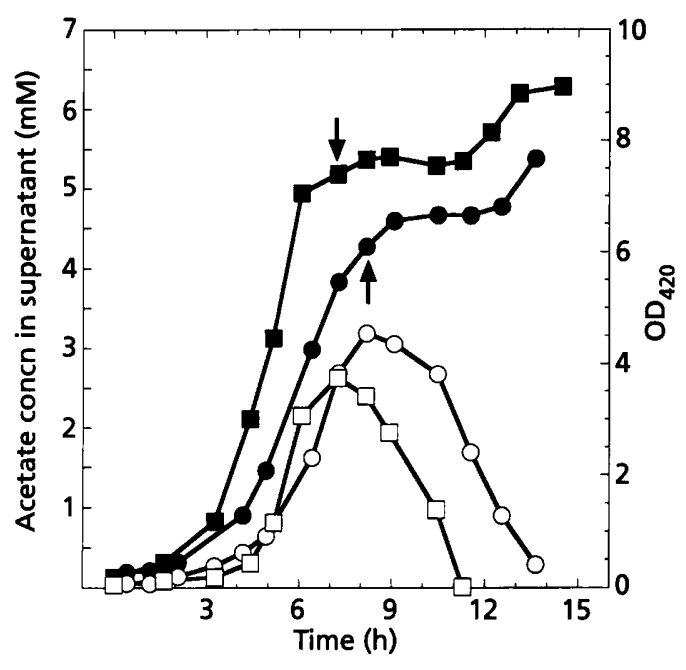

Fig. 3. Growth of $E$. coli MG1655 and mutant CWML2 on M9 medium containing $0.5 \%$ glucose as the growth-limiting substrate $\left(37^{\circ} \mathrm{C}, \mathrm{pH} 7.0 \pm 0.2\right.$, aeration 1.5 v.v.m.). $\mathrm{OD}_{420}$ of wildtype (O) and mutant CWML2 ( $\square$ ) is shown on the right axis; the left axis shows the acetate concentration in the supernatants of wild-type $(O)$ and mutant $(\square)$. Arrows indicate the time of complete depletion of glucose for each strain.

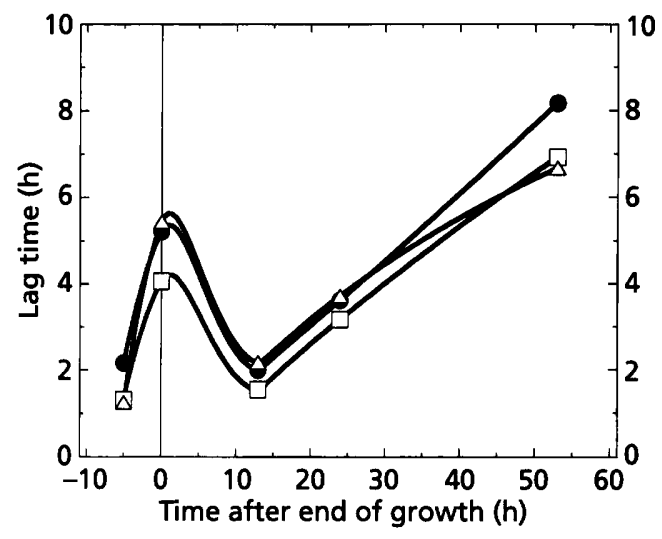

Fig. 4. Lag phase duration for the different mutants in M9 medium with glucose as a function of inoculum growth phase. Lag times are indicated for MG1655 (O), mutant CWML1 $(\triangle)$ and mutant CWML2 ( $\square$ ). The given values are means of eight determinations for each time point and strain. These derive from two independent batch cultures and four different determinations per culture.

on glucose was observed for mutant CWML2 (Fig. 3). Transient accumulation of lactate was greatly reduced in this mutant, but the maximum concentration of succinate was found to be twice as high as that of the wild-type. As for growth on glycerol, an increase in $\mathrm{OD}_{420}$ was observed for both mutants after depletion of glucose and all previously formed overflow products. Both mutants, particularly mutant CWML2, showed a significantly reduced specific carbon dioxide production rate during exponential growth on glucose (Table 1).

\section{Lag phase and stress resistance}

As a general observation, batch cultures of both mutants frequently appeared to enter exponential growth phase more rapidly than did those of the wild-type. To quantify this reduction in lag phase intervals, inocula from batch cultures were used for a growth assay in microtitre plates. Quadruplicate determinations in two independent experiments were used for each strain and time point. The duration of the lag phases followed a common pattern for all strains inspected. The longest lag phases were observed for inocula from cultures at the transition to stationary phase or late in the stationary phase ( $>40$ h) (Fig. 4). Mutant CWML2 showed significantly shorter lag phases in all cases. The behaviour of CWML1 closely resembled the wild-type, with the exception of inocula from the exponential and late stationary phases, which resumed growth more rapidly than the wild-type.

As the observed reduction in lag phase durations of both mutants might be related to a generally altered stress response, the stress resistance of mutants and wild-type was investigated in more detail. In order to distinguish between the stress resistant state commonly associated with stationary phase E. coli (Matin et al., 1989) and the stress resistance of growing cells, cells from both exponential and stationary growth phase were subjected to several stress conditions. The cell densities at the beginning of the incubations were $2 \times 10^{5}$ c.f.u. $\mathrm{ml}^{-1}$ for samples from the exponential phase and $10^{7}$ c.f.u. $\mathrm{ml}^{-1}$ for samples from stationary phase cultures. Generally, an improved stress resistance was observed for all cells harvested from stationary phase. This was most pronounced for survival under $1 \mathrm{M} \mathrm{NaCl}$ osmotic stress. Mutant CWML2 exhibited significantly improved resistance against all stress challenges investigated (Fig. 5), except for resistance to high osmolarity of stationaryphase-derived cells. Variations from the wild-type behaviour, with the exception of heat-shocked cells, were most pronounced in cells harvested from the exponential growth phase. In the case of heat shock, the difference was more pronounced for stationary-phase-derived cells. The behaviour of mutant CWML1 was more inconsistent than that of CWML2. In response to nutrient deprivation and osmotic stress, this mutant resembled the wild-type. Significantly improved survival was only observed during heat shock.

\section{DISCUSSION}

Previously reported communities of $E$. coli mutants were obtained from chemostat selection using glucose as the carbon source at intermediate dilution rates (Helling et al., 1987; Kubitschek, 1970; Novick \& Szilard, 1950). Displayed traits of polymorphism were stabilized by a combination of different adaptive strategies (Rosenzweig et al., 1994). Under these conditions, co-metabolism based on the formation and consumption of overflow metabolites was established. The major fraction of the population consisted of mutant cells showing higher uptake rates and affinity for glucose in com- 

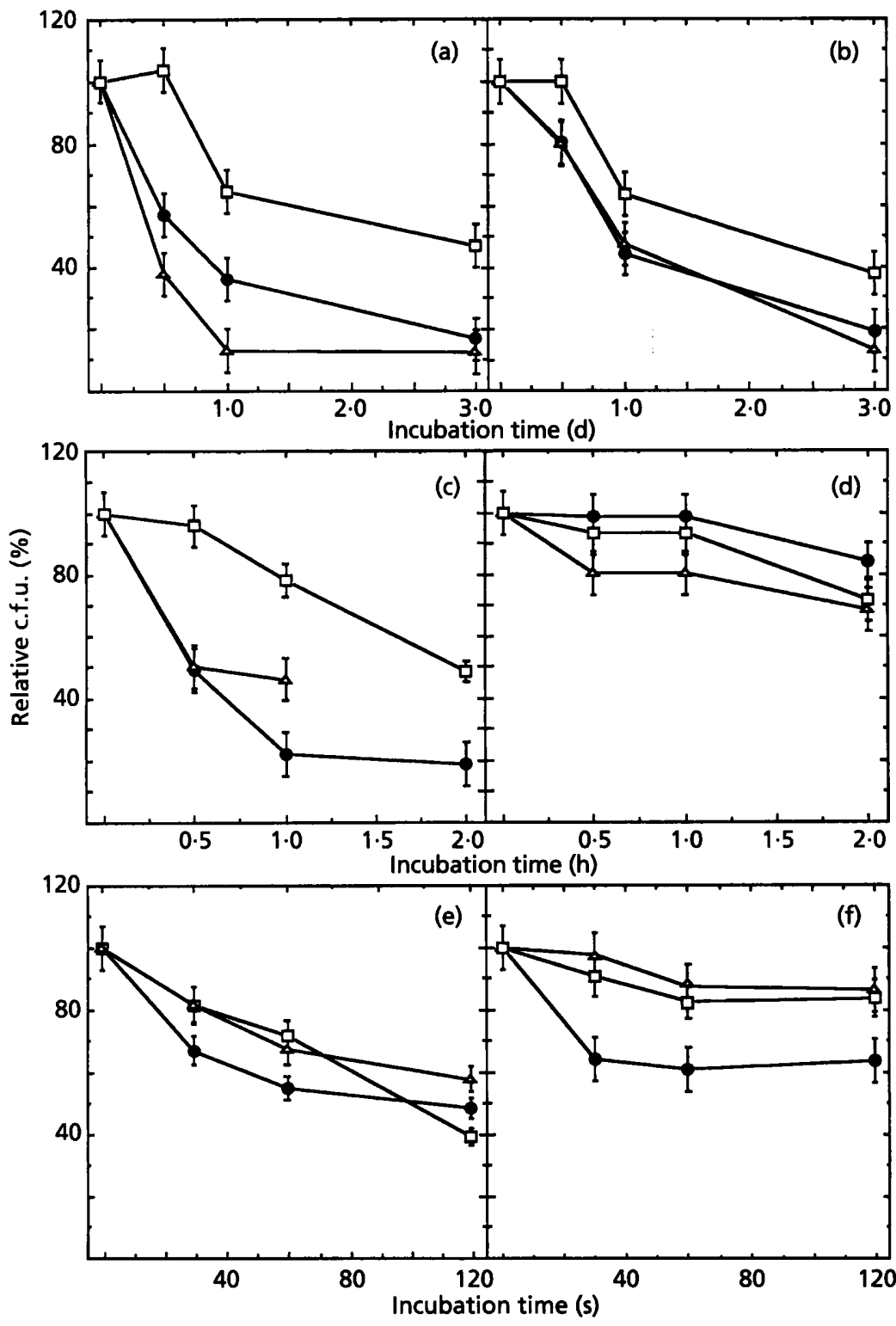

Fig. 5. Growth-phase-dependent resistance to carbon starvation, high osmolarity (1 M $\mathrm{NaCl})$ and heat stress $\left(55^{\circ} \mathrm{C}\right)$. The survival of wild-type (O), mutant CWML1 $(\triangle)$ and mutant CWML2 ( $\square$ ) cells is indicated. Graphs on the left $(a, c, e)$ show results for cells harvested from exponentially growing cultures, while those on the right $(b, d, f)$ show data for stationary phase cells. The responses to carbon starvation, high osmolarity and heat stress are shown in the first $(a, b)$, second $(c, d)$ and third $(e, f)$ row of graphs, respectively. Note the differing time scales. The data represent the mean c.f.u. value from two independent cultures per strain, with eight plates counted per time point. The $95 \%$ confidence intervals are given. bination with increased acetate excretion. Smaller fractions of specialized cells were selected to efficiently use the excreted acetate. Under the conditions used in our chemostat experiment, the substrate supply was relatively small, enough that no measurable overflow products were secreted by the growing cells. Consequently overflow products could not serve as an additional carbon source and thus did not constitute a selective pressure. No mutants with increased byproduct formation were found.

In our glycerol-limited continuous cultivation, E. coli mutants with different colony morphologies were obtained. This development of mutants was apparently the result of mutation and segregation of different populations during the selection experiment. In experiments with Pseudomonas aeruginosa, using glycerol as carbon source under similar conditions, mutants with increased substrate scavenging and utilization capacity were selected (Williams et al., 1994). In our selection, clones deriving from morphology $\mathrm{S}$ showed reduced growth rates in batch cultivations with glucose and acetate in combination with a strong tendency to adhere to walls, a behaviour previously described for bacterial chemostat selection systems (Contois, 1959). With respect to selection dynamics, the presence of these cells is probably of minor importance, since cells with morphology $S$ comprised only a small proportion of the total population. Clones deriving from morphology $\mathrm{L}$, the predominant morphology in the chemostat, generally exhibited higher growth rates and biomass yields in batch cultivations. In the mixed population evolved during the selection experiment, other strains besides the two mutant clones analysed were also present.

An interesting feature of the two strains obtained in this work is improvements in observed growth characteristics for carbon sources differing from that used for 
the selection. Apparently refuting the suggestion made by Rosenzweig et al. (1994) that the catabolic pathways for glucose, glycerol and acetate are coupled in a manner that precludes the creation of a single optimal strain, we were able to select for such a strain. The choice of the selective substrate has a seemingly strong influence on the nature of the altered catabolic capacities. Although neither of the isolated mutants was deficient in acetate production, they both produce either equal or reduced levels of acetate upon batch growth on glucose and glycerol. Reductions in acetate accumulation have been the focus of attempts to improve biomass and product yields in E. coli (Calhoun et al., 1993; Chou et al., 1994; Dedhia et al., 1994; Kakuda et al., 1994). A mutant with the acetate kinase gene deleted was impaired in growth on glucose but showed high rates of secretion and uptake of acetate, implying that this pathway is of little importance to the control of excess carbon flow (Kakuda et al., 1994). Mutant strains defective in acetate kinase and phosphotransacetylase were transformed to allow inducible glycogen overaccumulation and were shown to be a promising starting point for constructing $E$. coli strains with improved characteristics in high cell density fermentations (Dedhia et al., 1994).

Most interestingly, one of the isolated mutants displayed the biotechnologically desirable property of significantly improved robustness to adverse physiological conditions. When subjected to heat shock, osmotic stress or nutrient deprivation, exponentially growing or stationary phase cultures of mutant CWML2 generally exhibited increased viability compared to the wild-type. A significantly shortened lag phase was also observed for inoculated batch cultures of mutant CWML2. This was independent of the growth phase of the inoculum. Natural isolates of $E$. coli drastically increase their maximal growth rates to the level of laboratory strains when grown in a glucose-limited chemostat for 280 generations (Kurland, 1992). This transition to a laboratory strain phenotype was found to be combined with a partial loss of the ability to survive prolonged periods of nutrient shortage. Due to the low specific growth rate and the scarce substrate availability, our selection system apparently resulted in the generation of mutants with natural-isolate-like stress resistance which still maintain the high growth rate of laboratory strains.

Under the conditions used for selection, the chemostat culture was probably maintained at the transition between growth and stationary phase. Induction of stationary phase does not require cessation of growth. In particular, carbon-limited chemostat cultures of $E$. coli were reported to show extensive expression of stationary phase marker genes while growing at dilution rates below $0 \cdot 2 \mathrm{~h}^{-1}$ (Notley \& Ferenci, 1996). This transition phase is generally assumed to be characterized by low levels of cAMP (Chesbro et al., 1990) and high levels of ppGpp (Tedin \& Bremer, 1992). Glycerol as the sole carbon source increases the stringency of this regulation, as it inhibits protein synthesis in a manner similar to ppGpp (Svitil et al., 1993). Potentially as a result of altered stationary phase regulation, mutant CWML2 did not show the typical wild-type discrimination between exponential growth phase and stationary growth phase with respect to stress resistance. Thus it displays a combination of phenotypes at different growth phases. The described phenotype and the apparent changes at the protein level, as observed by twodimensional gel electrophoresis, suggest that the isolated strains are the result of at least one, but possibly more, pleiotropic mutations.

In this study, a foundation for the development of improved industrial biocatalysts has been established. A strain with improved properties of potential biotechnological interest has been isolated.

\section{ACKNOWLEDGEMENTS}

This research was supported by the Swiss Priority Program in Biotechnology (SPP BioTech). We thank Dr Daniel R. Lasko for critical reading of the manuscript.

\section{REFERENCES}

Bachmann, B. J. (1987). Derivations and genotypes of some mutant derivatives of Escherichia coli K-12. In Escherichia coli and Salmonella typhimurium: Cellular and Molecular Biology, pp. 1190-1219. Edited by F. C. Neidhardt, J. L. Ingraham, K. Brooks Low, B. Magasanik, M. Schaechter \& H. E. Umbarger. Washington, DC: American Society for Microbiology.

Calhoun, M. W., Oden, K. L., Gennis, R. B., Teixeira de Mattos, M. J. \& Neijssel, O. M. (1993). Energetic efficiency of Escherichia coli: effects of mutations in components of the aerobic respiratory chain. J Bacteriol 175, 3020-3025.

Cheng, C. Y., Yabe, I. \& Toda, K. (1989). Predominant growth of alpha-amylase regulation mutant in continuous culture of Bacillus caldolyticus. J Ferment Bioeng 67, 176-181.

Chesbro, W., Arbige, M. \& Eifert, R. (1990). When nutrient limitation places bacteria in the domains of slow growth: metabolic, morphologic and cell cycle behavior. FEMS Microbiol Ecol 74, 103-119.

Chou, C.-H., Bennett, G. N. \& San, K.-Y. (1994). Effect of modified glucose uptake using genetic engineering techniques on high-level recombinant protein production in Escherichia coli dense cultures. Biotechnol Bioeng 44, 952-960.

Contois, D. E. (1959). Kinetics of bacterial growth: relationship between population density and specific growth rate in continuous cultures. J Gen Microbiol 21, 40-50.

Dedhia, N. N., Hottinger, T. \& Bailey, J. E. (1994). Overproduction of glycogen in Escherichia coli blocked in the acetate pathway improves cell-growth. Biotechnol Bioeng 44, 132-139.

Dykhuizen, D. \& HartI, D. (1981). Evolution of competitive ability in Escherichia coli. Evolution 35, 581-594.

Dykhuizen, D. E. \& Hartl, D. L. (1983). Selection in chemostats. Microbiol Rev 47, 150-168.

Helling, R. B., Vargas, C. N. \& Adams, J. (1987). Evolution of Escherichia coli during growth in a constant environment. Genetics 116, 349-358.

Kakuda, H., Shiroishi, K., Hosono, K. \& Ichihara, S. (1994). Construction of a Pta-Ack pathway deletion mutant of Escherichia coli and characteristic growth profiles of the mutants in a rich medium. Biosci Biotechnol Biochem 58, 2232-2235. 
Kubitschek, H. E. (1970). Introduction to Research with Continuous Cultures. Englewood-Cliffs: Prentice-Hall.

Kurland, C. G. (1992). Translational accuracy and the fitness of bacteria. Annu Rev Genet 26, 29-50.

MacPhee, D. G. (1993). Directed mutations: a critical analysis. ASM News 59, 297-299.

Matin, A. (1992). Physiology, molecular biology and applications of the bacterial starvation response. J Appl Bacteriol Symp (Suppl.) 73, 49S-57S.

Matin, A., Auger, E. A., Blum, P. H. \& Schultz, J. E. (1989). Genetic basis of starvation survival in nondifferentiating bacteria. Annu Rev Microbiol 43, 293-316.

Moser, H. (1958). The Dynamics of Bacterial Populations Maintained in the Chemostat. Washington, DC: Carnegie Institute of Washington (Publication 61).

Notley, L. \& Ferenci, T. (1996). Induction of RhoS-dependent functions in glucose-limited continuous culture: what level of nutrient limitation induces the stationary phase of Escherichia coli. J Bacteriol 178, 1465-1468.

Novick, A. \& Szilard, L. (1950). Experiments with the chemostat on spontaneous mutations in bacteria. Proc Natl Acad Sci USA 36, $708-719$.

Paquin, C. \& Adams, J. (1983). Frequency of fixation of adaptive mutations is higher in evolving diploid than haploid yeast populations. Nature 302, 495-500.

Rosenzweig, R. F., Sharp, R. R., Treves, D. S. \& Adams, J. (1994). Microbial evolution in a simple unstructured environment: genetic differentiation in Escherichia coli. Genetics 137, 1-13.

Sakamoto, S., Terada, I., Lee, Y. C., Uehara, K., Matsuzawa, H. \& Ijima, M. (1996). Efficient production of Thermus protease aqualysin-1 in Escherichia coli: effect of cloned gene structure and 2-stage culture. Appl Microbiol Biotechnol 45, 94-101.

Svitil, A. L., Cashel, M. \& Zyskind, J. W. (1993). Guanosine tetraphosphate inhibits protein synthesis in vivo. J Biol Chem 268, 2307-2311.

Tedin, K. \& Bremer, H. (1992). Toxic effects of high levels of ppGpp in Escherichia coli are relieved by $r p o B$ mutations. J Biol Chem 267, 2337-2344.

Williams, S. G., Greenwood, J. A. \& Jones, C. W. (1994). The effect of nutrient limitation on glycerol uptake and metabolism in continuous cultures of Pseudomonas aeruginosa. Microbiology 140, 2961-2969.

Received 6 August 1996; revised 5 November 1996; accepted 17 December 1996. 